\title{
Search for Gravitino Dark Matter Decay with IceCube Data
}

\section{The IceCube Collaboration ${ }^{\dagger}$}

${ }^{\dagger}$ http://icecube.wisc.edu/collaboration/authors/icrc15_icecube

E-mail: japepperecrimson.ua.edu

The IceCube Neutrino Observatory has recently observed a flux of TeV-PeV neutrinos that corresponds to a highly significant excess over atmospheric backgrounds. It has been suggested that these events could originate from the decay of very heavy dark matter. We outline here an analysis of the IceCube data observed in 2011 with respect to the neutrino emission from heavy gravitino dark matter decay in the Galactic Halo.

Corresponding authors: J. Pepper ${ }^{1 *}$

${ }^{1}$ Department of Physics and Astronomy, University of Alabama, Tuscaloosa, AL 35487, USA

The 34th International Cosmic Ray Conference,

30 July- 6 August, 2015

The Hague, The Netherlands

${ }^{*}$ Speaker. 


\section{Introduction}

The observational evidence for Dark Matter (DM) is overwhelming, but its true nature remains elusive. One often invoked explanation comes in the form of Weakly Interacting Massive Particles (WIMPs) which have masses in the $\mathrm{GeV}-10 \mathrm{TeV}$ range. These particles are expected to accumulate in the center of massive celestial objects like the Earth, the Sun, galaxies or galaxy clusters. As these particles annihilate with each other or decay, an indirect detection is possible via the observation of secondary particles, such as gamma-rays, cosmic rays, or neutrinos.

IceCube is a cubic-kilometer neutrino detector installed in the ice at the geographic South Pole [1] between depths of $1450 \mathrm{~m}$ and $2450 \mathrm{~m}$. Detector construction started in 2005 and finished in 2010. Neutrino reconstruction relies on the optical detection of Cherenkov radiation emitted by secondary particles produced in neutrino interactions in the surrounding ice or the nearby bedrock. IceCube has previously conducted searches for WIMP annihilation in various astrophysical objects such as the Sun [2], Galactic Halo [3], and galaxy clusters [4].

However, it has been speculated [5-9] that the recent observed flux of extraterrestrial neutrinos with energies ranging from $30 \mathrm{TeV}$ to $2 \mathrm{PeV}[10,11]$ could originate from the decay of Very Heavy Dark Matter (VHDM). The observed abundance of DM can be reached if VHDM is produced nonthermally in the early Universe $[12,13]$. This type of DM could decay with lifetimes much longer than the age of the Universe, producing neutrinos in the same energy range as the high energy events observed by IceCube [5-9]. One such candidate for VHDM is a heavy gravitino [14]. Neutrinos from decaying gravitino dark matter should arrive with a unique energy spectrum and directional distribution proportional to the dark matter density of the galaxy.

\section{Simulation}

The observed flux of neutrinos from DM decay depends on the distribution of DM in the Milky Way, its lifetime, and decay spectrum. In the following we will focus on the decay of gravitino DM as outlined in [14] and consider decays into $\gamma \nu, Z v, h v$, and $W^{+} \tau^{-}$following the procedure outlined in [14]. Whereas the neutrino from the primary gravitino decay involving a neutral boson produces a line spectrum, the decay of the $Z$ and $W$ bosons as well as the Higgs $h$ and tau $\tau$ produces a continuum emission. We consider $100 \%$ branching into each decay channel, and model the decay with PYTHIA 6 [15]. The neutrino flux at IceCube resulting from gravitino dark matter is shown in Figure 1 for a dark matter mass of $2 \mathrm{PeV}$ and a lifetime of $10^{28} \mathrm{~s}$.

Simulated neutrino events in IceCube were weighted to represent the spectrum that would result from gravitino decay. This weighting was performed according to the equation for differential flux:

$$
\frac{d \Phi}{d E_{v}}=\frac{1}{\tau} \frac{R_{s c} \rho_{s c}}{4 \pi m_{\chi}} J(\psi) \frac{d N}{d E_{v}}
$$

where $\tau$ is the lifetime of the dark matter particle, and $d N / d E_{v}$ is the differential neutrino flux produced with PYTHIA. $R_{s c}$ and $\rho_{s c}$ represent the radius of the solar circle and the local dark matter density respectively. The dimensionless factor $J(\psi)$ corresponds to the integral over the line of sight of the dark matter density $\rho$ : 


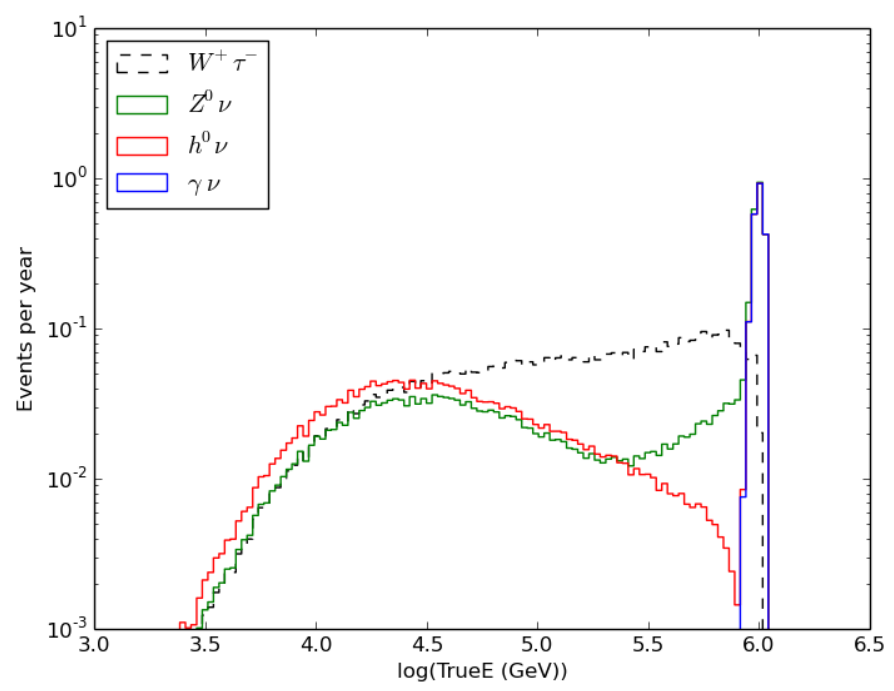

Figure 1: Neutrino spectrum at IceCube produced by $2 \mathrm{PeV}$ decaying gravitino dark matter assuming a lifetime of $10^{28} \mathrm{~s}$ and $100 \%$ branching into each decay mode.

$$
J(\psi)=\int_{0}^{l_{\max }} \frac{\rho\left(\sqrt{R_{s c}^{2}-2 l R_{s c} \cos (\psi)+l^{2}}\right)}{R_{s c} \rho_{s c}} d l
$$

with

$$
l_{\max }=\sqrt{R_{M W}^{2}-R_{s c}^{2} \cdot \sin ^{2}(\psi)+R_{s c} \cdot \cos (\psi)}
$$

where $R_{M W}$ is the extent of the Milky Way dark matter halo (40 kpc used in this analysis) and $\psi$ is the angular distance from the galactic center. For this analysis we use the Burkert dark matter profile, as parameterized in [16] to model the matter distribution in the Milky Way:

$$
\rho_{B u r}=\rho_{H} \frac{1}{\left(1+\frac{r}{R_{H}}\right)\left(1+\frac{r^{2}}{R_{H}^{2}}\right)}
$$

The initial neutrino flavor composition from the decay is not directly observable. One has to account for neutrino oscillation over cosmic distances that result in an oscillation-averaged flavor composition. In general, an arbitrary initial flavor ratio will lead to an oscillation average that is close to an equal distribution between all flavors [17]. For simplicity, we will assume in the following that each neutrino flavor in the gravitino decay spectrum carries $1 / 3$ of the total flux calculated by PYTHIA.

\section{Event Selection \& Reconstruction}

This analysis is based on events that have been previously selected by the High Energy Starting Event (HESE) analysis [10,11]. The sample consists of events with no more than three photoelectrons (p.e.) out of the first 250 recorded in an outer veto layer of optical modules, as described in [10]. This veto region includes the top $90 \mathrm{~m}$, bottom $10 \mathrm{~m}$, and outermost strings of the detector 
as well as a region of high dust concentration known as the "dust layer". Additionally, the event must also produce at least 6000 p.e. to ensure (to 99.999\%) that cosmic ray muons would produce enough light in the veto region to be excluded. The HESE sample after three years of observation consists of 36 events ( +1 coincident event removed from the sample), whereas only one event per year is expected from atmospheric backgrounds. Assuming an astrophysical $E^{-2}$ power-law spectrum, the excess has a significance of $5.7 \sigma[11]$.

In order to identify possible spectral features and an anisotropic event arrival distribution expected from gravitino decay in the Galactic Halo, it is necessary to measure the energy and direction of the neutrino flux as accurately as possible. To this end, IceCube has many different methods of reconstructing the energy, position, and direction of the incoming neutrino from the timing and amount of light deposited in the detector at various positions.

At energies in and above the $\mathrm{TeV}$ range, neutrinos primarily interact via deep-inelastic scattering, which produces a hadronic shower (cascade) at the interaction vertex. Charged-current interactions, where a charged boson is exchanged, produce a hadronic cascade accompanied by an outgoing lepton. This lepton can trigger an additional electromagnetic cascade in the detector, created by the outgoing electron, tau decay, muon bremsstrahlung, and/or pair production. All neutrino interactions produce cascades with the exception of $v_{\mu}$ charged-current interactions, which produce a hadronic cascade and an accompanying 'track' of light from the bremsstrahlung radiation of the outgoing muon.

The specific cascade reconstruction used in this analysis started with a basic line fit, which reconstructs a track under the assumption that the cascade vertex would lie somewhere along this track. Using this track as a seed, a cascade fitter positioned the vertex along this line to find the most likely position where the interaction occurred in the detector. The best fit vertex was then used as the seed for a more complex reconstruction algorithm which uses a likelihood method for fitting energy, direction, and position simultaneously in order to find the best fit values of these parameters [18].

\section{Binned Likelihood Analysis \& Sensitivity}

The neutrino flux from dark matter decay should be distinguishable from the atmospheric and astrophysical backgrounds by its unique energy spectrum and anisotropic arrival direction. While a limit could be set by counting the neutrinos in regions of energy and direction chosen so as to increase the signal to background ratio, greater sensitivity can be achieved by analyzing the overall shape of the reconstructed energy and direction simultaneously.

For a given two-dimensional histogram of data with respect to reconstructed energy and direction, the event distribution in each bin follows a Poisson distribution. The likelihood is then simply the product of Poisson probabilities for each bin, representing the total probability that the data was produced by the hypothesis. For a given distribution of data, the best-fit hypothesis is the one that maximizes the likelihood.

In order to calculate the sensitivity, the reconstructed energies and directions were binned with bin sizes equal to the width of the resolutions (Figure 2). Pseudodata was generated by sampling of Poisson distributions in each bin, with means given by the particular hypothesis. Using this pseu- 

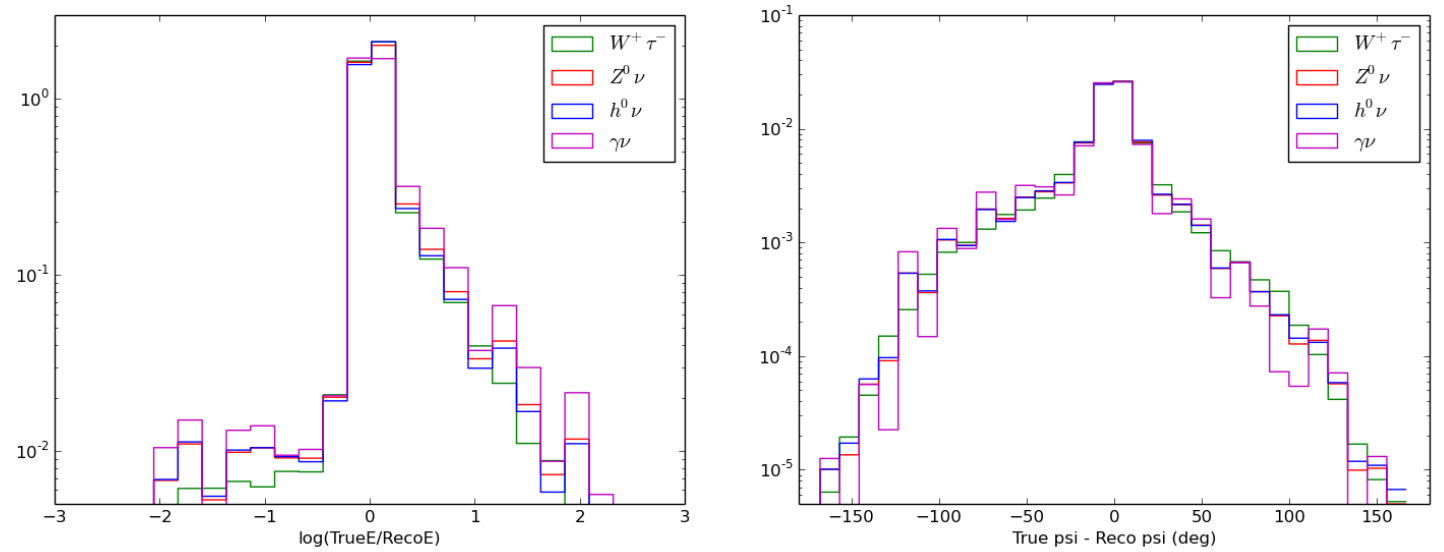

Figure 2: Left: Normalized histogram of the energy resolution for the two-body decay modes of a $2 \mathrm{PeV}$ gravitino. The standard deviation was used as the bin size in $\log (\mathrm{E})$ for the binned-likelihood sensitivity calculation. Right: Similarly to the energy resolution, this plot shows the distribution of angular resolution around the galactic center for a $2 \mathrm{PeV}$ gravitino. Optimal binning for this analysis is currently under study.

dodata, the best fit background-only hypothesis was compared to the best fit background+signal hypothesis using the test statistic (TS):

$$
\begin{array}{r}
\Lambda=\frac{\mathscr{L}_{\text {sig }+b g}}{\mathscr{L}_{b g}} \\
T S=-2 \cdot \ln (\Lambda)
\end{array}
$$

For this analysis, three likelihoods are considered (described below), which differ only in their interpretation of the background. For each likelihood, pseudodata representing background only sources as well as pseudodata representing background plus a given dark matter signal were generated 1000 times each. For each pseudoexperiment, a test statistic was calculated. This resulted in a distribution of test statistics for background-only pseudodata as well as a distribution of test statistics for background with injected signal. If $90 \%$ of the signal+bg TS distribution is above the median of the background-only TS distribution, the detector is said to be sensitive to that signal.

In the first likelihood method, the HESE power law fit to the astrophysical flux $\left(\Phi_{\text {astro }} \propto\right.$ $E^{-2.3 \pm 0.3}$ ) is considered to be the background along with neutrinos produced in the atmosphere. The astrophysical flux was allowed to vary within the HESE uncertainties to result in the best fit. This interpretation leads to a sensitivity/limit for the case where none of the events were caused by dark matter decay.

For the second method, the astrophysical background is assumed to be a power law with an unknown spectral index and normalization. This background is then allowed to take on the values which lead to the best fit. This interpretation allows for events to have been created by both the astrophysical flux as well as dark matter decay, and sets the sensitivity/limit accordingly.

Lastly, the third likelihood considers only atmospheric background, and $100 \%$ of the events 
detected are assumed to be from dark matter decay. The resulting sensitivities for each likelihood method are shown below in Table 1. As expected, decay modes with spectral lines perform better due to the distinct shape of their energy distribution with respect to background sources. The gravitino decay into $\gamma v$ gives slightly worse sensitivity than decay into $Z^{0} v$ or $h^{0} v$, which is currently thought to be due to the lack of neutrinos at lower energies.

Table 1: Preliminary sensitivities for $2 \mathrm{PeV}$ gravitino DM using the three likelihood methods

\begin{tabular}{|l|c|c|c|}
\cline { 2 - 4 } \multicolumn{1}{c|}{} & \multicolumn{3}{c|}{ Lifetime Sensitivity } \\
\hline Decay Mode & HESE flux + DM & Power law + DM & DM only \\
\hline$W^{+}+\tau^{-}$ & $10^{27.8} \mathrm{~s}$ & $10^{27.6} \mathrm{~s}$ & $10^{27.6} \mathrm{~s}$ \\
\hline$Z^{0}+v$ & $10^{27.9} \mathrm{~s}$ & $10^{27.8} \mathrm{~s}$ & $10^{27.8} \mathrm{~s}$ \\
\hline$h^{0}+v$ & $10^{27.9} \mathrm{~s}$ & $10^{27.7} \mathrm{~s}$ & $10^{27.8} \mathrm{~s}$ \\
\hline$\gamma+v$ & $10^{27.9} \mathrm{~s}$ & $10^{27.8} \mathrm{~s}$ & $10^{27.8} \mathrm{~s}$ \\
\hline
\end{tabular}

\section{Conclusions}

The decay of very heavy dark matter is an interesting hypothesis of the recently observed flux of high-energy neutrinos by IceCube. We have outlined our analysis to study this decay via a maximum likelihood test taking into account the energy and arrival direction of events. For all decay modes, the resulting lifetime sensitivities are approximately $10^{28} \mathrm{~s}$, with the most sensitive decay modes being the ones that produce spectral lines. The next step for this analysis will be to set a limit on the gravitino lifetime using the Feldman-Cousins method for DM masses in the PeV - $10 \mathrm{PeV}$ range.

\section{References}

[1] A. Achterberg et al., Astropart. Phys. 26 (2006) 155.

[2] M. G. Aartsen et al. [IceCube Collaboration], Phys. Rev. Lett. 110, 131302 (2013)

[3] R. Abbasi et al. [IceCube Collaboration], Phys. Rev. D 84, 022004 (2011).

[4] M. G. Aartsen et al. [IceCube Collaboration], Phys. Rev. D88 (2013) 122001 (2014).

[5] Arman Esmaili and Pasquale Dario Serpico, JCAP 1311 (2013) 054 [arXiv:1308.1105 [hep-ph]].

[6] Arman Esmaili, Sin Kyu Kang, and Pasquale Dario Serpico, JCAP 12 (2014) 054 [arXiv:1410.5979 [hep-ph]].

[7] Brian Feldstein, Alexander Kusenko, Shigeki Matsumoto, and Tsutomu T. Yanagida, Phys. Rev. D 88, 015004 (2013) [arXiv:1303.7320 [hep-ph]].

[8] Joachim Kopp, Jia Liu, and Xiao-Ping Wang, JHEP 04 (2015) 105 [arXiv:1503.02669 [hep-ph]].

[9] Jesus Zavala Phys. Rev. D 89, 123516 (2014) [arXiv:1404.2932 [astro-ph.HE]]. 
[10] M. G. Aartsen et al. [IceCube Collaboration], Science 342, 1242856 (2013) [arXiv:1311.5238 [astro-ph.HE]].

[11] M. G. Aartsen et al. [IceCube Collaboration], arXiv:1405.5303 [astro-ph.HE].

[12] M. Cirelli, Pramana 79, 1021 (2012), 1202.1454.

[13] A. Ibarra, D. Tran, and C. Weniger, Int.J.Mod.Phys. A28, 1330040 (2013), 1307.6434.

[14] M. Grefe, arXiv:1111.6779 [astro-ph.HE].

[15] T. Sjöstrand, S. Mrenna, and P. Skands, JHEP 05 (2006) 026 [hep-ph/0603175].

[16] F. Nesti and P. Salucci, arXiv:1304.5127 [astro-ph.GA].

[17] M. G. Aartsen et al. [IceCube Collaboration], Phys. Rev. Lett. 114, 171102 (2015).

[18] M. G. Aartsen et al. [IceCube Collaboration], arXiv:1311.4767 [astro-ph.HE]. 